
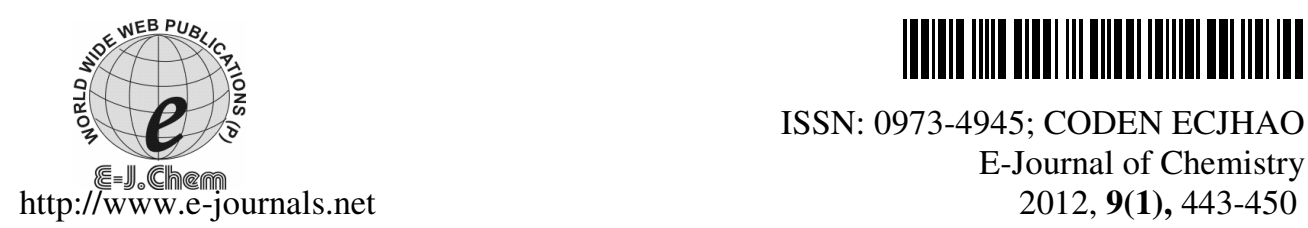

ISSN: 0973-4945; CODEN ECJHAO

E-Journal of Chemistry 2012, 9(1), 443-450

\title{
Micellar Liquid Chromatography for the Determination of Some Less Prescribed Benzodiazepines
}

\author{
HOONKA SUBHRA $^{\S *}$, BOSE DEVASISH $^{\S}$, \\ ESTEVE-ROMERO JOSEP ${ }^{\#}$ and DURGBANSHI ABHILASHA
}

Department of Crim. and Forensic Sciences

Dr. H.S.Gour University, Sagar, India

\#Àrea de Química Analítica

Universitat Jaume I, 12080, Castelló, Spain

Department of Applied Chemistry, Institute of Technology

Banaras Hindu University, Varanasi (U.P.). India

abhilashabose_12@rediffmail.com

Received 19 November 2010; Accepted 30 December 2010

\begin{abstract}
A simple chromatographic procedure is reported for the determination of some less prescribed but equally important benzodiazepines (Clotiazepam, clozapine and pinazepam) in serum. The optimization studies have been made in $\mathrm{CN}, \mathrm{C}_{18}$ and $\mathrm{C}_{8}$ columns, using mobile phase containing sodium dodecyl sulphate (SDS) modified with either propanol, butanol or pentanol. The method proposed for the determination of the three benzodiazepines using a mobile phase of $0.13 \mathrm{M}$ SDS, $2.4 \%$ pentanol$0.01 \mathrm{M}$ phosphate buffer- $0.1 \%$ triethylamine $(\mathrm{pH} 7)$ at $25^{\circ} \mathrm{C}$ and UV detection $(240 \mathrm{~nm})$ in $\mathrm{a}_{8}$ column. The serum samples was injected directly, without any pretreatment, eluted in less than $8 \mathrm{~min}$, in accordance to their relative polarities, as indicated by their octanol-water partition coefficients. The limits of detection (ng/mL) was in the 1.6 to 5.6 and 7 to 87 range, for aqueous and serum samples, respectively. Repeatability and intermediate precision was tested for three different concentrations of the drugs, resulting in the 0.1 to 2 range. The results obtained here for the separation of the three benzodiazepines in serum were also counter checked at Department of Bio-analytical Chemistry, Universitat Jaume I, Castelló, Spain.
\end{abstract}

Keywords: Micellar liquid chromatography, Benzodiazepines, Serum

\section{Introduction}

The introduction of chromatographic techniques in analytical toxicology in the late 1950s represented the first key moment in the evolution of systematic toxicological analysis (STA). Since that time it became possible to detect the presence of drugs in biological 
samples from its behavior in a given chromatographic system, provided that the same drug had been previously analyzed in that system and its retention behavior has been recorded ${ }^{1}$. One of the most important groups of drugs for monitoring is benzodiazepines. The Benzodiazepines cause sedation, hypnosis, decreased anxiety, muscle relaxation and some of them also possess anticonvulsant activity. In short these are the most important class of drugs used widely for the treatment of anxiety related condition. These drugs affect activity at all level of the neuraxis, though some structures are affected more than the others. All benzodiazepines are qualitatively similar in their effects on the important sleep parameters ${ }^{2}$. Administration of benzodiazepines to the mother before delivery causes apnoeic spells, hypotonia and hypothermia (floppy baby syndrome). They are often abused by the young illicit drug user and may lead to profound behavioral effects ${ }^{3,4}$. Benzodiazepines are known to cause dependence and addiction with tolerance and withdrawal effects in some patients. The older population is also more likely to develop significant adverse CNS effects from the use of benzodiazepines and it also present with an increased risk of motor vehicle crash. Because of a significant risk of agranulocytosis (in which the white blood cell count drops and increases the risk of infection), a potentially life-threatening adverse event, clozapine should be reserved for use in the treatment of severely ill schizophrenic patients who fail to show an acceptable response to adequate courses of standard antipsychotic drug treatment. Patients being treated with clozapine must have a baseline white blood cell (WBC) and differential count before initiation of treatment as well as regular WBC counts during treatment and for four weeks after discontinuation of treatment.

Because of their wide abuse, benzodiazepines are included in most drug abusescreening program. The determination of these less preferred benzodiazepines i.e. clotiazepam, clozapine and pinazepam is important as they have important biological properties which depend on their accurate concentration in blood. While benzodiazepines can be measured using a variety of techniques their structural diversity and wide variations in potency provide a challenge for laboratories to detect all relevant numbers in one analytical scheme.

Reviews of the analysis of benzodiazepines have been published ${ }^{5-8}$. Several other analytical methods are described in the literature for the screening and quantitation of benzodiazepines. From the first approaches with paper and thin-layer chromatography ${ }^{9,10}$ we have witnessed within a few years a rapid development of different chromatographic techniques, such as gas chromatography with the introduction of capillary columns (GC) $)^{11,12}$ and high performance liquid chromatography (HPLC) ${ }^{13,14}$ particularly with the introduction of the reversed phase mode, that have found widespread application in benzodiazepines. Presently GC coupled to a mass spectrometer (MS) is considered as the method of choice for a confirmation $^{15,16}$. Indeed this is a highly sensitive and specific method, which identifies drug without ambiguity, but the equipment and maintenance are relatively costly and need qualified operators. Other chromatographic methods have been developed for the identification of benzodiazepines in biological samples: GC coupled with electron capture, flame ionization detector and reversed phase liquid chromatography coupled with UV detector ${ }^{17,18}$.

Analysis of serum samples for drugs presents special problems because of the high and variable protein content. If proteins are not completely removed from blood serum, they may result in a poor resolution of otherwise easily determined sample constituents. The protein retained on the column leads to high backpressure and finally blocking the column, which completely destroys the separation. In view of this, it is advisable to design an extraction procedure for biological matrix that does not allow small amount of disturbing components, 
such as proteins, to be left in the sample matrix. Although sample pretreatment or protein precipitation can eliminate matrix effects, it is not suitable for routine analysis in the clinical laboratories because it adds to the total analysis time, the risk of contamination and the risk of sample loss.

Owing to the capability of SDS to solubilize proteins, serum samples can directly be injected into the column using micellar liquid chromatography (MLC). MLC is a variant of RP-HPLC in which the mobile phase is composed of a surfactant in a concentration above the critical micellar concentration. In some cases a modifier, such as 1-propanol, 1-butanol, 1-pentanol, is added to decrease the retention factor and increase the efficiencies. The direct injection of serum samples in MLC allows rapid analysis, which can be performed on very small sample volumes. Our goal was to achieve separation conditions that could permit complete resolution of benzodiazepines from endogenous components of serum matrix. These conditions permitted direct injection of plasma samples without the need for sample pretreatment prior to MLC analysis.

\section{Experimental}

The reagents used in the mobile phases were: the surfactant sodium dodecyl sulphate (99\% purity, Merck, India), the modifiers 1-propanol, 1-butanol, 1-pentanol (BDH, India) and the buffer compounds sodium dihydrogenphosphate and $\mathrm{HCl}$ or $\mathrm{NaOH}$ (Ranchem, India). Triethylamine and isopropylamine was purchased from Merck, India. The mobile phases were filtered through $0.45 \mu \mathrm{m}$ Nylon membranes (Micron Separations, Westboro, MA, USA). Methanol (BDH, India) was used to condition the column.

The analytes were: pinazepam (Tedec-Meiji Farma, Madrid), clotiazepam and clozapin were from novartis Pharmaceuticals (Barcelona, Spain). The above drugs were kindly donated by the cited pharmaceutical companies in Spain. Stock solutions containing $100 \mu \mathrm{g} / \mathrm{mL}$ were prepared by dissolving the compounds in a few milliliters of methanol, with the aid of an ultrasonic bath. The water used was double distilled water. The serums in blank and with the drug used for the analysis were kindly donated by the local pathology, Sagar, India and were stored below $0{ }^{\circ} \mathrm{C}$ in refrigerator.

\section{Apparatus}

The $\mathrm{pH}$ of the mobile phases was measured with a systonic, India, $\mathrm{pH}$ meter, provided with a combined $\mathrm{Ag} / \mathrm{AgCl} / \mathrm{glass}$ electrode. UV spectra and absorbance measurements were obtained with a Shimadzu UV-Vis spectrophotometer, Shimazdu, Japan. Maximum wavelengths and molar absorptivities of the drugs were recorded using the above spectrophotometer.

A Beckman chromatograph was used for the separation, which was provided with an isocratic pump, manual injector, a UV-visible detector set at $240 \mathrm{~nm}$. The signal was acquired by a PC computer connected to the chromatograph. Measurement of peak properties and optimization of mobile phase composition were assisted by the MICHROM software. The columns used for the analysis were, C-18, CN and Kromasil C-8 (Scharlau, Spain, $5 \mu \mathrm{m}$ particle size, $250 \mathrm{~mm} \times 4.6 \mathrm{~mm}$ i.d.). The flow-rate used was $1 \mathrm{~mL} / \mathrm{min}$ and the injection volume was $20 \mu \mathrm{L}$.

\section{Chromatographic analysis}

The composition of the micellar mobile phase recommended for the determination of the drugs studied in this work is: $0.13 \mathrm{M}$ SDS, 2.4\% pentanol, $0.01 \mathrm{M}$ sodium dihydrogenphosphate and $0.1 \%$ triethylamine. The $\mathrm{pH}$ was adjusted at 7 with $\mathrm{HCl} / \mathrm{NaOH}$ before the addition of the modifiers. 
The serum sample used was spiked with the benzodiazepines. The sample solutions should be filtered before injection into the chromatograph. The standard solutions of the drugs were also filtered. Filtration was always performed into the vials through $0.45 \mu \mathrm{m}$ Nylon membranes of $13 \mathrm{~mm}$ diameter.

\section{Results and Discussion}

\section{Nature of the modifier}

The polarities of benzodiazepines are low. Consequently, their retention on a $\mathrm{C}_{8}$ column was excessive when eluted with pure micellar mobile phases of SDS, and after the addition of the modifiers methanol or propanol, even at high concentrations. Elution at appropriate retention times was finally achieved by the addition of an alcohol with a longer chain, such as butanol or pentanol. There are references in the MLC literature on the use of pentanol as a modifier in analytical procedures. This alcohol permitted the elution of diverse hydrophobic compounds, such as steroids (0.1 M SDS-7\% pentanol mobile phase $)^{20}$ and sulphonamides (0.05 M SDS-2.4\% pentanol mobile phase $)^{21}$. Butanol has not been employed before in MLC analytical applications, but it has been compared with other modifiers in physicochemical studies ${ }^{22,23}$.

Figure 1 shows a contour map of the mobile phase containing SDS as surfactant and pentanol as modifier. The figure shows that at the lowest concentration of SDS and pentanol there is no resolution as is evident form the contour at $0.05 \mathrm{M} \mathrm{SDS}$ and $2 \%$ pentanol. As we increase the concentration of pentanol and keep the SDS concentration fixed at $0.05 \mathrm{M}$, efficiency increases but the resolution is not satisfactory. Therefore as observed in figure 1 the best resolution can be achieved somewhere in the range of 0.11-0.14 M SDS and 2-3\% of pentanol.

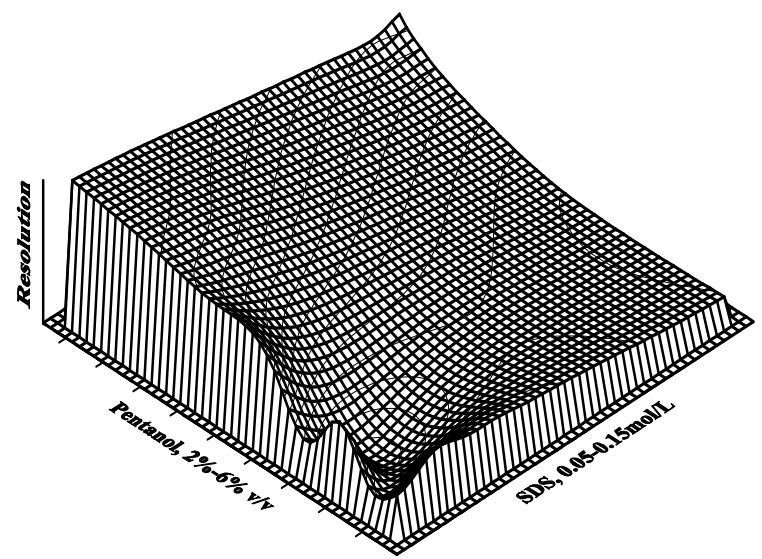

Figure 1. Contour map of resolution for three benzodiazepines eluted with SDS-pentanol mobile phase

Pentanol yielded better efficiencies and lesser retentions than butanol. The peaks of all the three benzodiazepines could also be resolved with butanol but with more analysis time. pentanol was thus preferred to optimize the separation of the three drugs.

pH selection

No reference was found in the literature on the protonation constants of the studied drugs, but for other benzodiazepines at least two acid-base equilibria with $\log K_{l} 8-11$ and $\log K_{2} 2-3$ 
have been reported ${ }^{2} . \log K_{2}$ is expected to increase in the presence of the anionic SDS micelles, owing to stabilization of the positive charge of the protonated drugs. Thus, at $\mathrm{pH} 3$, benzodiazepines should be partially protonated at the N-4 position.

A series of measurements were carried out with $0.13 \mathrm{M} \mathrm{SDS}-2.4 \%(\mathrm{v} / \mathrm{v})$ pentanol mobile phases, where the $\mathrm{pH}$ was varied between 3 and 7 . The retention was effectively somewhat larger below $\mathrm{pH} 4$ for clozapine and pinazepam. Owing to the greater over-lapping of these two compounds at $\mathrm{pH} 4-5$, mobile phases were next buffered at $\mathrm{pH} 7$.

\section{Concentrations of surfactant, alcohol and amine}

The elution behavior of the benzodiazepines was usual in MLC: an increase in the concentration of surfactant resulted in decreased retentions and efficiencies and an increase in the concentration of alcohol modifier yielded decreased retentions but enhanced efficiencies. Due to the low polarity of the drugs, the efficiencies were however still low with added alcohols. The elution strength for pentanol was greater than for SDS, in the 1-6\% pentanol and 0.05-0.15 M SDS concentration ranges.

Amines, such as triethylamine (TEA) ${ }^{24}$ and isopropylamine ${ }^{25}$, have been added to the mobile phase in some analytical RPLC procedures proposed for benzodiazepines. The use of an amine is a common practice to protect the silanol groups of the stationary phase, in order to increase peak efficiencies for compounds having amine groups. The effect of TEA on the separation of the drugs was thus examined. The concentration of TEA was varied in the $0-0.2 \%$ concentration range for a $0.13 \mathrm{M} \mathrm{SDS}-2.4 \%$ pentanol mobile phase buffered at $\mathrm{pH} 7$. The addition of TEA enhanced the efficiencies for these compound. However, the amine behaved as another modifier and the retention factors of the compounds were reduced. For this reason, the concentration of TEA was limited to $0.1 \%$.

Using the resolution diagram for SDS-pentanol mobile phases, resulting that $0.13 \mathrm{M}$ SDS-2.4\% $(v / v)$ pentanol, $0.1 \%$ TEA and $\mathrm{pH} 7$ is the most adequate for giving good resolution, low analysis time and highest efficiencies. In this mobile phase the elution order was (min): pinazepam (5.0), clotiazepam (6.2), clozapine (7.3). For individual determination of the three benzodiazepines, Figure 2 shows the mobile phases that give analysis time of 8 min using pentanol.

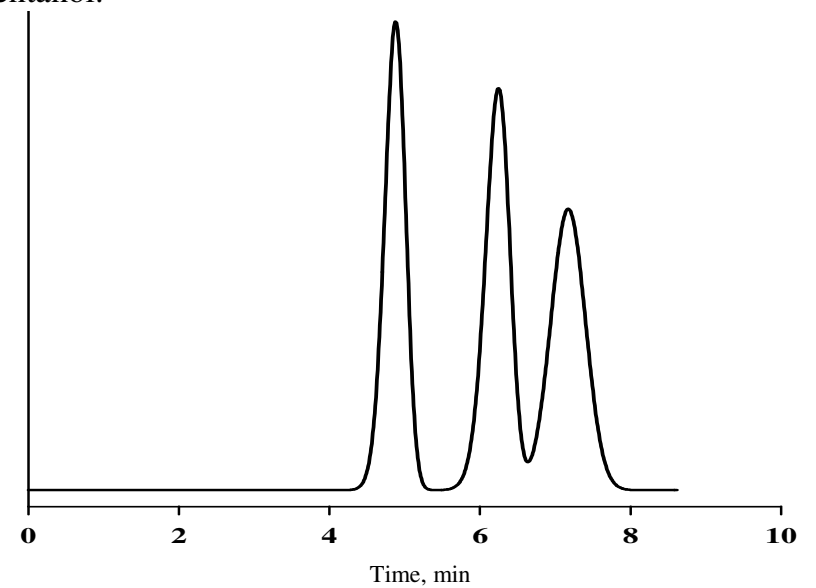

Figure 2. Chromatogram of pinazipam, clotiazepam and clozapine eluted with 0.13 M SDS$2.4 \%$ pentanol. 


\section{Linearity and detection limit}

Calibration curves were constructed for each benzodiazepine, using the measured areas of the chromatographic peaks at six increasing concentrations in the range from 1 to $50: \mathrm{g} \mathrm{mL}^{-1}$ in spiked serum samples. The slopes, intercepts and regression coefficients of the calibration curves were in satisfactory range (Table 1).

Table 1. Calibration parameters and limits of detection (LODs in $\mathrm{ng} \mathrm{mL}^{-1}, 3$ s criterium) for the determination of three benzodiazepines using the optimum mobile phase SDS130 mM $2.4 \%(v / v)$ pentanol-pH 7 and ultraviolet detection at $240 \mathrm{~nm}$

\begin{tabular}{ccccc}
\hline Sample & Slope & Intercept & $r$ & LOD \\
\hline Pinazepam & 86.17 & +0.560 & 0.9999 & 0.3 \\
Clotiazepam & 72.07 & +1.887 & 0.9999 & 0.3 \\
Clozapine & 59.18 & -0.228 & 0.9999 & 0.4 \\
\hline
\end{tabular}

Limits of detection (LODs, 3s criterion) were evaluated by the injection of series of 10 solutions, containing the benzodiazepines at the lowest concentration of the calibration curve. The LODs were between 1.6 to $5.6 \mathrm{ng} / \mathrm{mL}$ and 7 to $87 \mathrm{ng} / \mathrm{mL}$ range, for aqueous and serum samples, respectively. The values of the LODs were similar to those usually reported in the literature and permitted the detection and quantification of the benzodiazepines in serum with the method proposed in this work, taking into account that the serum samples can be injected without any previous treatment.

\section{Repeatability and intermediate precision}

Repeatability values were calculated by measuring the areas of the peaks obtained by injection of series of ten spiked serum samples with three different concentrations of benzodiazepines (Table 2). The injections were performed in the calibration range indicated above. The variation coefficient was always below $1.8 \%$. The intermediate precision was also obtained and the variation coefficients of the peak areas in samples injected during five different days were in the $0.1-2 \%$ range.

\section{Analyses of benzodiazepines in spiked serum samples}

The background signal of serum, due to proteins (wide band in the head of the chromatograms) and several endogenous compounds (peaks at diverse retention times) can seriously affect the detection of the benzodiazepines. Direct injection of spiked serum samples (without any dilution) mainly made the detection difficult for the substances that are less retained. However, dilution of the spiked serum samples before its injection reduced the width of the protein band and of some endogenous peaks, allowing the detection of the drugs.

Table 2. Intra and interday precision (RSD in $\%, n=10$ ) using three different concentrations, of three benzodiazepines $\left(c_{1}=600, c_{2}=300\right.$ and $\left.c_{3}=150 \mathrm{ng} \mathrm{mL}{ }^{-1}\right)$ using the optimum mobile phase SDS130 mM -2.4\% ( $v / v)$ pentanol-pH 7 and ultraviolet detection at $240 \mathrm{~nm}$

\begin{tabular}{ccccccc}
\hline \multirow{2}{*}{ Sample } & \multicolumn{3}{c}{ Intra-day } & \multicolumn{3}{c}{ Inter-day } \\
\cline { 2 - 7 } & $\mathrm{c}_{1}$ & $\mathrm{c}_{2}$ & $\mathrm{c}_{3}$ & $\mathrm{c}_{1}$ & $\mathrm{c}_{2}$ & $\mathrm{c}_{3}$ \\
\hline Pinazepam & 0.22 & 0.66 & 0.80 & 0.20 & 0.43 & 0.56 \\
Clotiazepam & 0.34 & 0.89 & 1.11 & 0.46 & 0.8 & 0.95 \\
Clozapine & 0.24 & 0.58 & 0.97 & 0.54 & 1.07 & 1.60 \\
\hline
\end{tabular}


Moreover, the injection of a large number of serum samples can produce damage to the packing material, thus shortening the life of the column, or can force a frequent regeneration of the stationary phase. It was therefore decided to carry out the analysis of serum samples after their dilution. In these conditions, retention times were unchanged at least after 250 injections into the chromatographic system. For all the benzodiazepines studied, the sensitivity achieved after dilution in a 1:10 factor was adequate for their detection in serum.

Figure 3(A) shows the chromatograms in the MLC optimum mobile phase of blank serum sample while Figure 3(B) is the chromatogram of spiked serum samples containing the individual benzodiazepines. The results indicate that the MLC procedure can be easily used for the determination of three benzodiazepines (pinazepam, clozapine and clotiazepam) in serum samples with analysis time below 8 min using a mobile phase of $0.13 \mathrm{M} \mathrm{SDS}-2.4 \%$ pentanol, $0.1 \%$ TEA at $\mathrm{pH}$ 7. The procedure is sensitive enough to monitor these drugs in serum at the therapeutic and toxicological levels, taking into account that the serum sample was injected without any previous treatment.

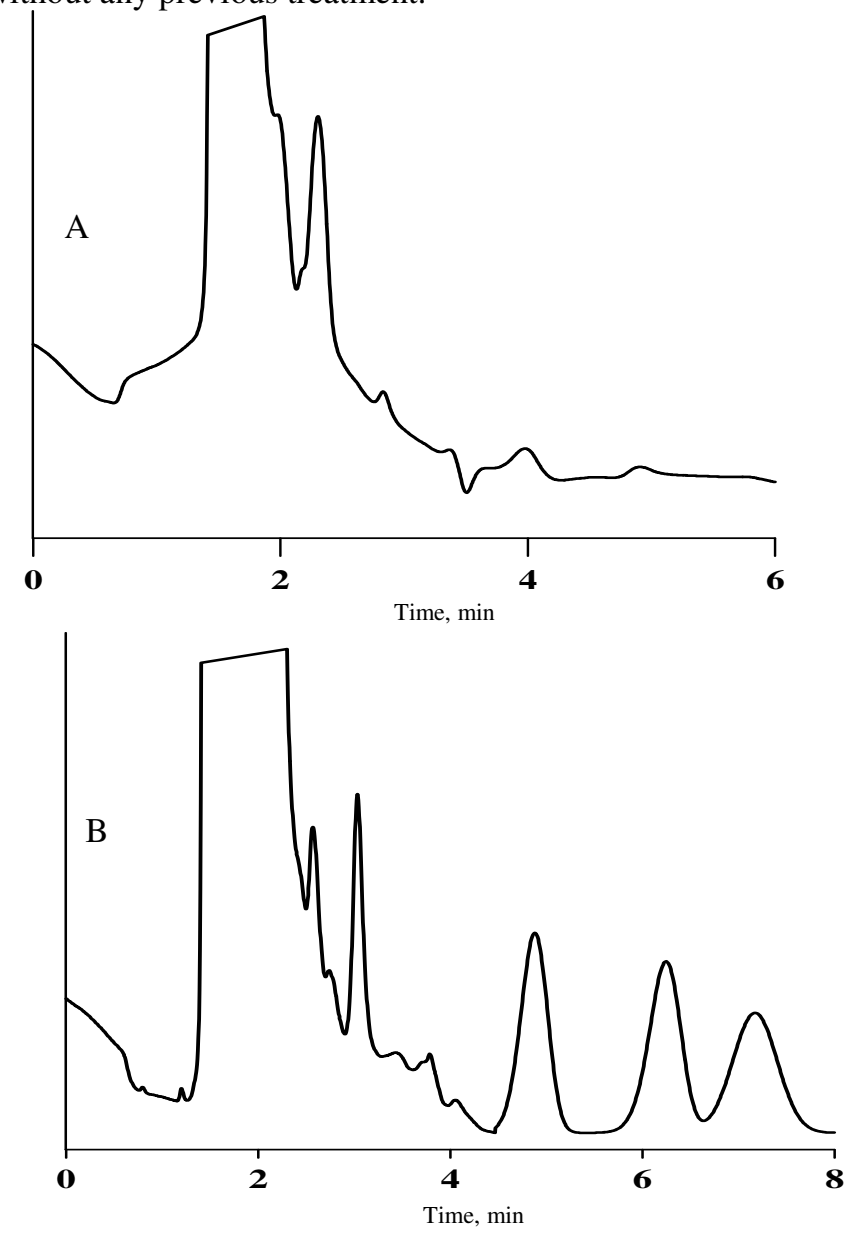

Figure 3. (A) Chromatogram of blank serum sample eluted in optimum mobile phase (B) Chromatogram of spiked serum sample containing three benzodiazepines in optimum mobile phase 


\section{Acknowledgement}

Dr. Abhilasha Durgbanshi thanks Council of Scientific and Industrial Research for providing Research Associateship and Dr. Devasish Bose thanks Directorate of Forensic Science, Ministry of Home Affairs, Government of India for providing research grant. Ms. Subhra Hoonka thanks University Grants Commission, Government of India, for providing Junior Research Fellowship.

\section{References}

1 Kovats E, Helv Chim Acta, 1958, 41, 1915-1932.

2 Bethesda M D, American Hospital Formulary Service, American Society of the board of health-System Pharmacists, 1998.

3 Drummer O H and Ranson D L, Am J Forensic Med Pathol., 1996, 17(4), 336-342.

4 Zweigenbaum J, Heinigk K, Steinborner S, Wachs T and Henion J, Anal Chem., 1999, 71, 2294-2300.

5 Drummer O H, J Chromatogr B., 1999, 733, 27.

6 Drummer O H, J Chromatogr B., 1998, 713, 201.

$7 \quad$ Maurer H and Pfleger K, J Chromatogr., 1987, 422, 85.

8 Maurer H H, J Chromatogr., 1992, 580, 3-41.

9 Tames F, Watson I D, Morden W E and Wilson I D, J Planar Chromatogr Mod TLC, $2000,13,432$.

10 Higvegi E, Perneczki S and Forstner M, J Planar Chromatogr Mod TLC., 2000, 13, 414.

11 Guan F, Seno H, Ishii A, Watanabe K, Kunazawa T, Hattori H and Suzuki O, J Anal Toxicol., 1999, 23(1), 54-61.

12 Ulrich M L and Sagar F, Beitr-Gerichtl-Med., 1988, 46, 135-141.

13 Kagan M Z, J Chromatogr A, 2001, 918, 293.

14 El Mahjoub A and Staub C, J Chromatogr B, 2001, 754, 271.

15 Paterson S, Mclachlan-Troup N, Cordero R, Dohnal M and Carman S, J Anal Toxicol., 2001, 25(3), 203-208.

16 Soriano T, Jurado C, Menendez M and Rapetto M, J Anal Toxicol., 25, 2001, 137-143.

17 Kunick P K, J Chromatogr B: Biomed Sci Appl., 2001, 750, 41.

18 Ferrara S D, Tedeschi L, Frison G and Catagna F, J Anal Toxicol., 1992, 16, 217-222.

19 Torres-Lapasió J R, Berthod A and García-Alvarez-Coque M C, Ed., Micellar Liquid Chromatography (Marcel Dekker, New York), 2000.

20 Torres-Cartas S, García-Alvarez-Coque M C and Villanueva-Camanas R M, Anal Chim Acta, 1995, 302, 163-172.

21 García-Alvarez-Coque M C, Simó-Alfonso E F, Ramis-Ramos G and EsteveRomero J S, J Pharm. Biomed Anal., 1995, 13(3), 237-245.

22 García M A, Vera S, Bombín M and Marina M L, J Chromatogr., 1993, 646, 297.

23 Rodríguez-Delgado A, Sánchez M A, González V and García-Montelongo F, Chromatogr., 1994, 38, 342.

24 Le-Solleu L, Demotes-Mainard F, Vincon G and Bannwarth B, J Pharm Biomed Anal, 1993, 11(8), 771-775.

25 Lambert W E, Meyer E, Xue-Ping Y and De-Leenheer A P, J Anal Toxicol., 1995, 19(1), 35-40. 


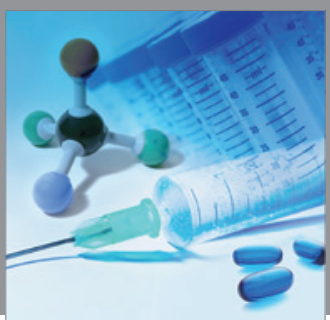

International Journal of

Medicinal Chemistry

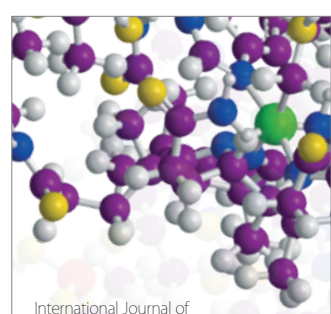

Carbohydrate Chemistry

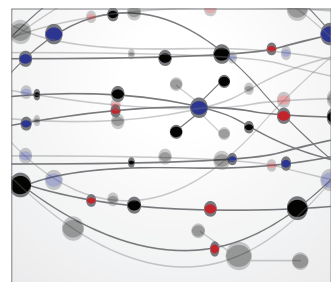

The Scientific World Journal
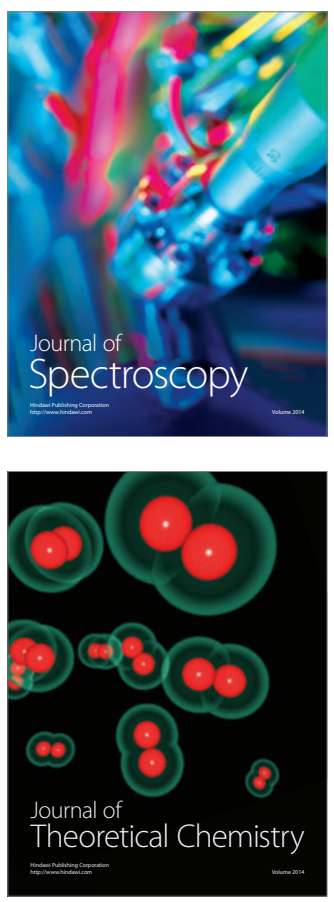
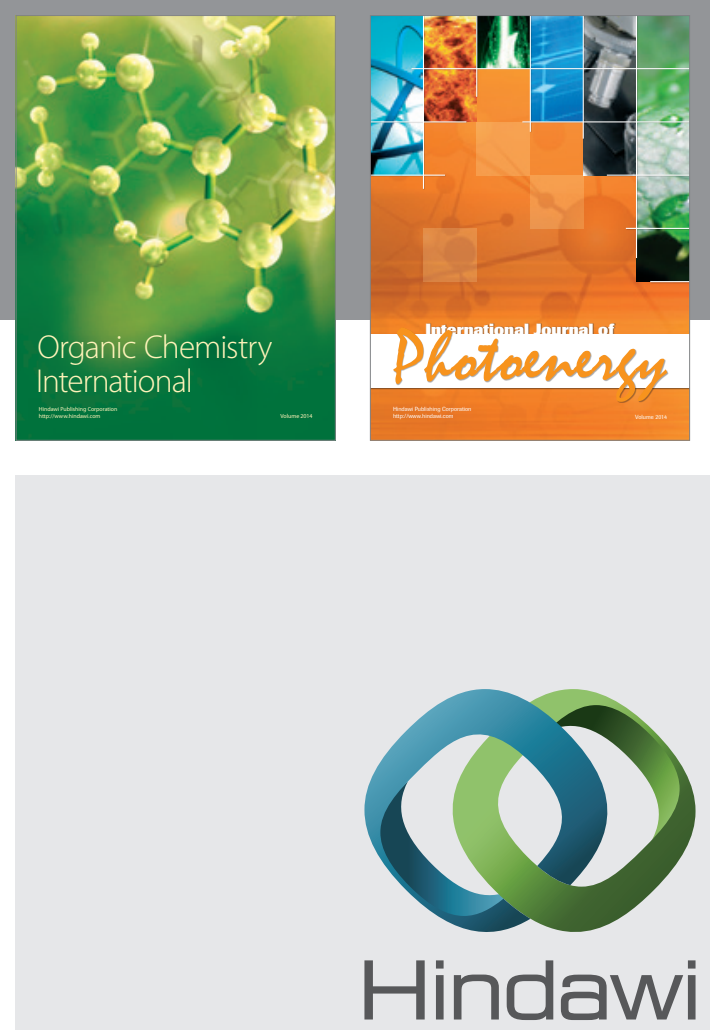

Submit your manuscripts at

http://www.hindawi.com
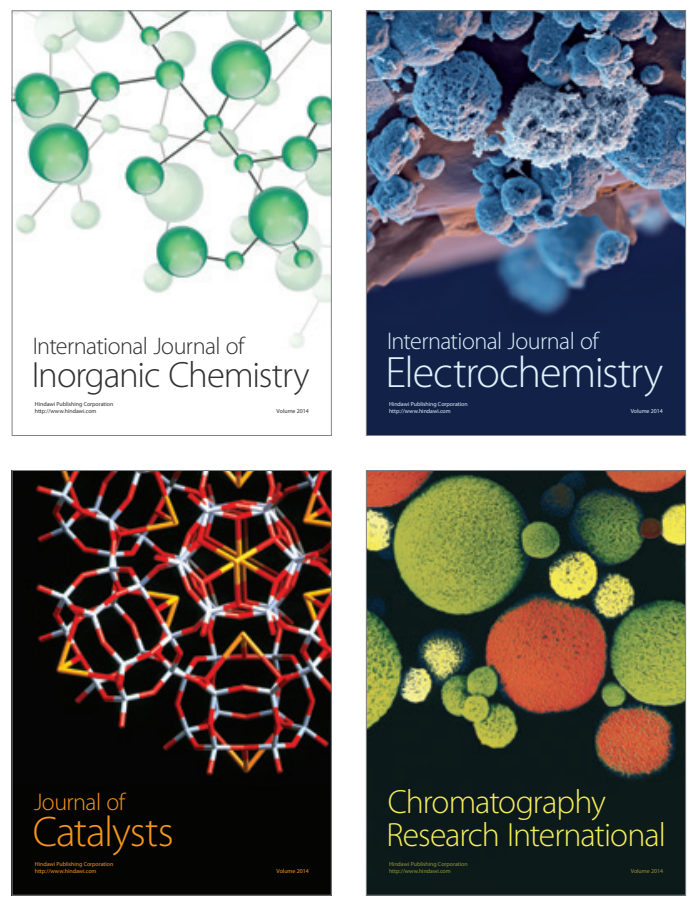
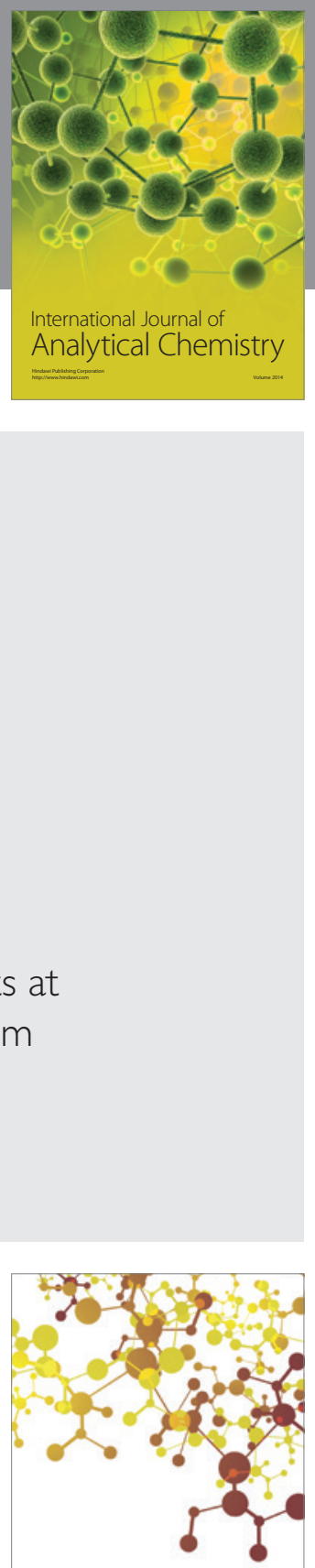

Journal of

Applied Chemistry
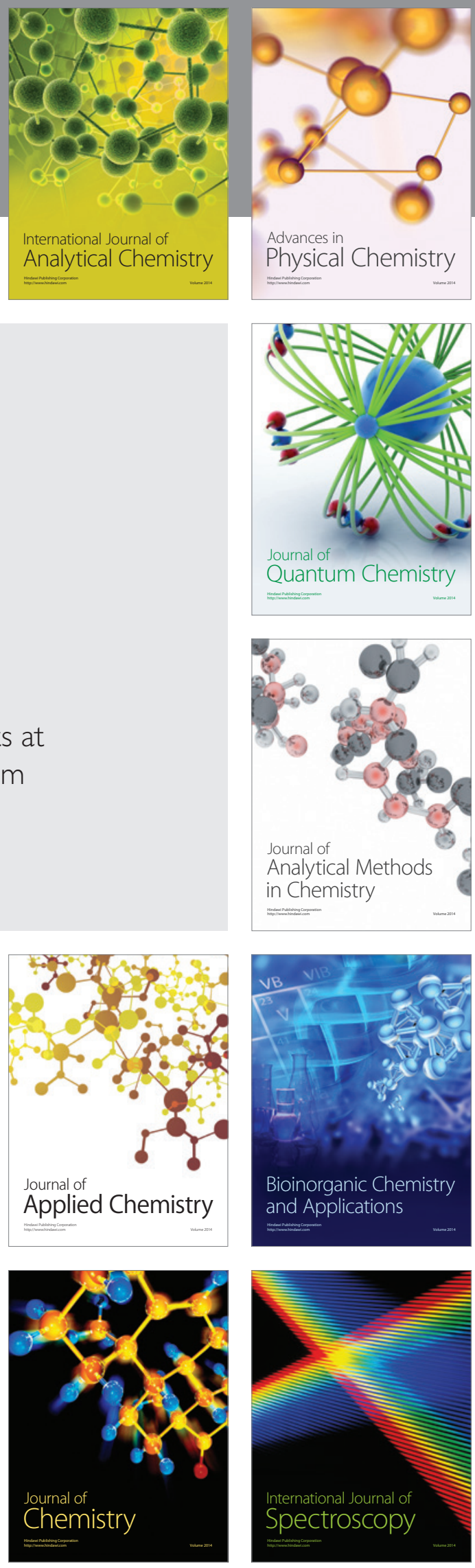